\title{
Cellular types in acute leukaemia: diagnosis and significance ${ }^{1}$
}

\author{
F. W. GUNZ AND A. F. BURRY \\ From the Departments of Pathology, Christchurch, and Princess \\ Margaret Hospitals, Christchurch, New Zealand
}

SYNOPSIS Two independent observers, one using haematological and the other histological methods, analysed material from 68 patients with acute leukaemia. A correct diagnosis of the cell type of leukaemia was made in $70 \%$ of cases by haematological methods, and in $83 \%$ of those cases histological examination gave the same result. In two-thirds of the haematologically equivocal cases histological examination provided a positive diagnosis. The finally undiagnosed cases amounted to $12 \%$ of the total. The types so diagnosed could not be correlated with any clinical features.

Differentiation of the cell types in acute leukaemia showed that children with granulocytic types of acute leukaemia survived for shorter periods than those with other types but in adults the type of cell had no significance when related to survival. In adults the immediate prognosis for all cell types was relatively bad. The unfavourable prognosis attached to a high initial leucocyte count was confirmed.

The diagnosis of leukaemia is made by haematological and clinical means, and its differentiation into acute and chronic forms presents as a rule no serious obstacles. By contrast, the further subdivision of acute leukaemia into cellular types is subject to considerable uncertainty, for there are few universally agreed diagnostic criteria. Such distinctions as may be drawn appear often to represent the views of individual observers rather than an analysis of objectively verifiable facts. In the words of a recent review: 'Morphology is more an art than a science and ... the morphologic distinction between lymphoblasts and myeloblasts is better seen than described' (Boggs, Wintrobe, and Cartwright, 1962). Such an approach to diagnosis may procure reproducible results from the members of a single school but its shortcomings become evident when statistics from several sources are compared, for only a lack of generally accepted criteria can explain the gross differences in distribution of types reported, sometimes from neighbouring centres (Gunz and Hough, 1956). The authors of the Seventh Revision of the International List of Causes of Death have realistically accepted these difficulties by merely providing one single category (204.3) for all

\footnotetext{
${ }^{1}$ This investigation was supported in part by research grant no. C-3707 from the National Cancer Institute, U.S. Public Health Service.
}

Received for publication 2 January 1963. forms of acute leukaemia, except acute monocytic leukaemia and erythraemia (Di Guglielmo's disease). For purposes of international comparison, acute leukaemia thus appears as a single disease.

The question has been argued whether a subdivision of acute leukaemia is of more than academic interest. Clinically, many cases have indeed a fairly uniform course, and few distinctive features have been recognized, other than the widely accepted predominance of the lymphocytic type in childhood. The introduction of new forms of treatment has, however, shown a need for a re-assessment of this question, since there are reports from several authors that the various types may respond differentially to a chemotherapeutic approach (Haut, Altman, Wintrobe, and Cartwright, 1959; Freireich, Gehan, Sulman, Boggs, and Frei, 1961; Boggs et al., 1962), while others have found factors such as the patient's age of greater importance (Ellison, 1956; Louis, Sanford, and Limarzi, 1958). The investigation now to be reported was made with the double aim of ascertaining if any objective criteria enabled acute leukaemias to be separated into types, and whether haematological or pathological differences between such groups could be correlated with their clinical behaviour. If such were found to be the case, it appeared possible that the results could be used for improving the accuracy of prognosis in acute leukaemia. 


\section{MATERIAL AND METHODS}

The plan of the investigation was to examine critically, by means of a combined haematological and histological approach, the validity of some criteria currently used for the differential diagnosis of acute leukaemias. For this purpose the available material was studied by two independent observers, one of whom was responsible for the initial haematological and the other for the histological diagnosis. Neither observer was acquainted with the names or clinical particulars of any of the patients or with their presumed diagnoses, all cases being strictly studied as 'unknown' by both.

The cases came from our own hospitals, as well as from many other centres throughout New Zealand, and had been obtained in the course of a survey into the incidence, type distribution, and aetiology of leukaemia in a total circumscribed population between 1958 and 1962. A total of 359 cases of acute leukaemia had been reported under this scheme, but post-mortem material (which was essential for our purpose) was available from only 79 cases. In 11 of these either the haematological or histological material was incomplete, so that the final study series consisted of 68 cases. Although we did not select material, except according to availability, the series was not a random one, since its members were preponderantly adults. This arose from the fact that relatively few necropsies were carried out on children with acute leukaemia, either because they did not die in hospitals or for other reasons. The overall percentage of necropsies in the whole series was 44 ; this included patients dying at home as well as in hospitals.

At the beginning of the investigation each case was given an index number which remained its only identification until the final assessment. Detailed criteria (listed below) were laid down for the analysis of the haematological and histological appearances, and the findings, measured by these criteria, were entered on standard work-sheets. In many instances two separate 'blind' examinations of individual cases were carried out on different dates. Haematological and histological diagnoses were then made where possible and compared with each other. Both observers then made a further examination of the material from all cases in which the initial diagnoses differed, the material being again coded. Finally all cases were classified either as diagnosed or undiagnosed, and the clinical data were inspected and correlated with the pathological ones.

The material which we analysed had been obtained for routine diagnostic purposes and prepared by standard methods. Blood and marrow smears were treated by one of the Romanowsky and sometimes a peroxidase stain. Histological sections were stained by haematoxylin and eosin and sometimes by silver impregnation. No material was specially treated for this investigation. Our findings are thus based on observations which could have been made in any diagnostic laboratory provided the same set of criteria had been used for the evaluation of the appearances. This general applicability of our results was an essential purpose of the investigation.

The following criteria were used in the haematological examination of all blood and marrow films: General appearance, quality and cellularity of films; presence and relative number of neutrophil polymorphonuclears, myelocytes, lymphocytes, monocytes, and other blood or marrow cells (no differential counts were made); nature of $\vec{\Rightarrow}$ blasts, as follows: size of cell relative to erythrocytes and its outline, shape of nucleus, size of nucleus relative to whole cell, presence of more than one nucleus per cell, nuclear chromatin pattern, number and size of nucleoli, frequency of mitoses; staining characteristics of cytoplasm, presence of granules, vacuoles, of cytoplasmic inclusions. A general comment was then made on the $\mathrm{c}$ findings in each slide, and a diagnosis was made $\vec{\sigma}$ where possible in one of the following categories: granulocytic, lymphocytic, monocytic, eosinophilic, and Di Guglielmo's disease.

During the histological examination the presence or absence of leukaemic infiltration was noted in all organs sectioned, and particular attention was paid to the liver, spleen, and lymph nodes. The cytological character of the infiltrations was defined within the limitations of the $N$ technique, but the final diagnosis was based on the histological and not the cytological appearances of the sections. The following points relating to the site of infiltration were thought to be of especial significance.

LIVER If infiltration was seen predominantly or exclu- $\vec{\bullet}$ sively in the sinusoids, this was taken to favour a diagnosis $\mathrm{E}$ of granulocytic leukaemia; if mainly in the portal tracts, of lymphocytic leukaemia.

SPLEEN Infiltration of the red pulp with partial or complete preservation of Malpighian bodies was believed to be characteristic of granulocytic leukaemia; if the $\stackrel{\mathbb{\Omega}}{2}$ Malpighian bodies were diminished in size but could be $\overrightarrow{\vec{\overrightarrow{ }}}$ identified at all, lymphocytic leukaemia was held unlikely. An enlargement of the Malpighian bodies at the expense of the red pulp favoured a diagnosis of lymphocytic leukaemia.

LYMPH NODES Complete loss of structure of the whole or a segment of a node was the essential criterion for a 3 . diagnosis of acute lymphocytic leukaemia. Preferential involvement of the sinusoids and pulp was taken to $\frac{3}{3}$ result from an extrinsic process or from proliferation of sinusoidal epithelium and favoured the diagnosis of $\frac{}{2}$ granulocytic or monocytic leukaemia.

CYTOLOGICAL ASSESSMENT This was made on the strength of cellular size, amount of cytoplasm, and shape and vesicularity of the nucleus. The presence of cytoplasmic $N$ granules could not usually be determined, except in a few cases in which a prominent eosinophilic infiltration was present.

\section{RESULTS}

DEFINITE INITIAL HAEMATOLOGICAL DIAGNOSIS A diagnosis of the type of acute leukaemia was made $\mathbb{\mathbb { D }}$ in 47 cases $(69 \%)$ as a result of the examination of $\frac{?}{\mathbb{D}}$ blood and marrow films alone. Fifteen of these $\frac{\varrho}{\sigma}$ were examined as unknowns on two separate 
occasions, and the two diagnoses agreed in each case. There were 24 males and 23 females. The diagnoses were granulocytic 39, eosinophilic one, Di Guglielmo three, lymphocytic four.

Of all the haematological criteria used in the attempted classification by far the most useful was the presence of signs pointing to a transition of the blasts to more mature members of one or the other cell series. Thus many cases classed as granulocytic showed some degree of cytoplasmic granulation in at least a proportion of the primitive cells; others had a predominance of early myelocytes. The few cases of lymphocytic leukaemia showed an unbroken transition from blasts via immature to mature lymphocytes.

In a number of cases there were blasts containing no granules but definite Auer bodies (Table I). These were classified as granulocytic, as were those which showed a 'pseudo-Pelger' abnormality of the nuclei of myelocytes and polymorphonuclears (Dameshek and Gunz, 1958; Dorr and Moloney, 1959). The other cellular and nuclear appearances, such as size, shape, and nuclear chromatin pattern, were found to be of very little help in coming to a decision on the type present. In the great majority of cases the leukaemic cells were grossly atypical;

TABLE I

REASONS FOR DIAGNOSING ACUTE GRANULOCYTIC LEUKAEMIA

\begin{tabular}{lr} 
Reason & \multicolumn{1}{c}{ No. } \\
\hline Transition to more mature cells & $28(58 \%)$ \\
Predominance of promyelocytes & $3(6 \%)$ \\
Auer bodies & $4(8 \%)$ \\
Pseudo-Pelger & $4(8 \%)$ \\
Histology & $9(19 \%)$ \\
Total & $48(99 \%)$
\end{tabular}

such features as cellular and nuclear anisocytosis, irregularity of outline, presence of pseudopodia, lobe formation, or splitting of the nucleus were quite common and could not be correlated with the type. Similarly both number and size of the nucleoli were variable. In cases eventually classified as lymphocytic the number of nucleoli tended not to exceed three, but in those classified as granulocytic the range was very wide, both individually and over the whole series.

HiSTOLOGY OF haEMATOLOGICALLY DEFINITE CASES Independent histological examination produced the same diagnosis in 39 of the 47 cases in which a definite haematological diagnosis had been made. This was $83 \%$ of all haematologically 'definite' cases, and $57 \%$ of the total. In the other eight cases either a different or no diagnosis was made.
Cytological characteristics were more difficult to assess in sections than in haematological smears. The size of cells could be misleading as some acute granulocytic leukaemias showed a predominantly small blast cell with a dense hyperchromatic nucleus. As in the haematological assessment the best cytological criterion was a 'shading off' from pure blast through stages of myelocyte. By this means about half the granulocytic leukaemias could be diagnosed. Nuclear shape was not very helpful, as in the absence of demonstrable granules in routine sections immature myelocytes and monocytes could not be distinguished. The solitary case of eosinophilic leukaemia of course was easily diagnosed.

The histological appearances in lymph nodes were almost invariably clear cut although infiltration in acute granulocytic leukaemia was sometimes so extensive that follicles were barely discernible as a last remnant of node structure. Infiltration of nodes by megakaryocytes was sometimes a striking feature in granulocytic leukaemia.

In the spleen the findings were clear cut with the exception of two cases of granulocytic leukaemia where infiltration was so extreme that no vestige of the white pulp could be recognized in the sections examined. Goldberg in a series of papers (Goldberg, 1957; Goldberg, 1958; Goldberg and Ungar, 1958; Goldberg and Rubenstone, 1960; Goldberg, Rubenstone, and Saphir, 1961a and b) studied the deep lymphatic system of the spleen and claimed that all leukaemias could be classified into myelogenous and lymphogenous groups on the basis of the degree of infiltration of the deep lymphatic system. However, in several cases accepted without hesitation by us both as granulocytic, heavy infiltration of the subendothelial plexus of trabecular veins was seen, a finding which Goldberg claims is typical of lymphocytic leukaemia.

The histology of the liver was rarely by itself diagnostic, as also stated by Popper and Schaffner (1957). In lymphocytic leukaemia the portal tracts showed a variable infiltration with a characteristic sharp outline, while in granulocytic leukaemia their outline was irregular in shape and infiltrating cells shaded into the sinusoids which always showed heavy infiltration if the portal tracts were enlarged. In other organs no distinctions could be established by patterns of infiltration.

Table II compares the divergent haematological and histological diagnoses in the haematologically definite cases and lists the probable reasons for the differences. It is seen that the histological interpretations differed from the haematological ones either because the material was insufficient for a conclusive statement, or because the appearances were so atypical, sometimes, it seemed, because of extensive 
TABLE II

CASES WITH DIFFERING INITIAL HAEMATOLOGICAL AND HISTOlOGICAL DIAGNOSES

\begin{tabular}{|c|c|c|c|c|c|c|}
\hline No. & $\operatorname{Sex}$ & $\begin{array}{l}\text { Age } \\
\text { (yr.) }\end{array}$ & $\begin{array}{l}\text { Haematological } \\
\text { Diagnosis }\end{array}$ & $\begin{array}{l}\text { Reason for Haematological } \\
\text { Diagnosis }\end{array}$ & Histological Diagnosis & Reason for Difference \\
\hline $\begin{array}{l}19 \\
20\end{array}$ & $\begin{array}{l}\mathbf{F} \\
\mathbf{M}\end{array}$ & $\begin{array}{r}75 \\
7\end{array}$ & $\begin{array}{l}\text { Granulocytic } \\
\text { Granulocytic }\end{array}$ & $\begin{array}{l}\text { Transition to myelocytes } \\
\text { Transition to myelocytes }\end{array}$ & $\begin{array}{l}\text { ? Lymphosarcoma } \\
\text { ? Lymphocytic } \\
\text { ? Granulocytic }\end{array}$ & $\begin{array}{l}\text { Lack of complete material } \\
\text { Lack of complete material }\end{array}$ \\
\hline 29 & $\mathbf{F}$ & 64 & Granulocytic & Pseudo-Pelger & ? Hodgkin's disease & $\begin{array}{l}\text { Much fibrosis (case of post-radiatio } \\
\text { leukaemia) }\end{array}$ \\
\hline 42 & $\mathbf{M}$ & 65 & Granulocytic & Transition to myelocytes & No leukaemia & Heavy chemotherapy \\
\hline 54 & $\mathbf{M}$ & 16 & Granulocytic & Transition to myelocytes & ? Monocytic & Fibrosis after heavy chemotherapy \\
\hline 63 & $\mathbf{M}$ & 78 & Granulocytic & Transition to myelocytes & ? Monocytic & Atypical appearances \\
\hline 74 & $\mathbf{F}$ & 15 & Lymphocytic & Transition to lymphocytes & Granulocytic & Atypical appearances \\
\hline
\end{tabular}

TABLE III

CASES WITH INITIAL INDEFINITE HAEMATOLOGICAL DIAGNOSES

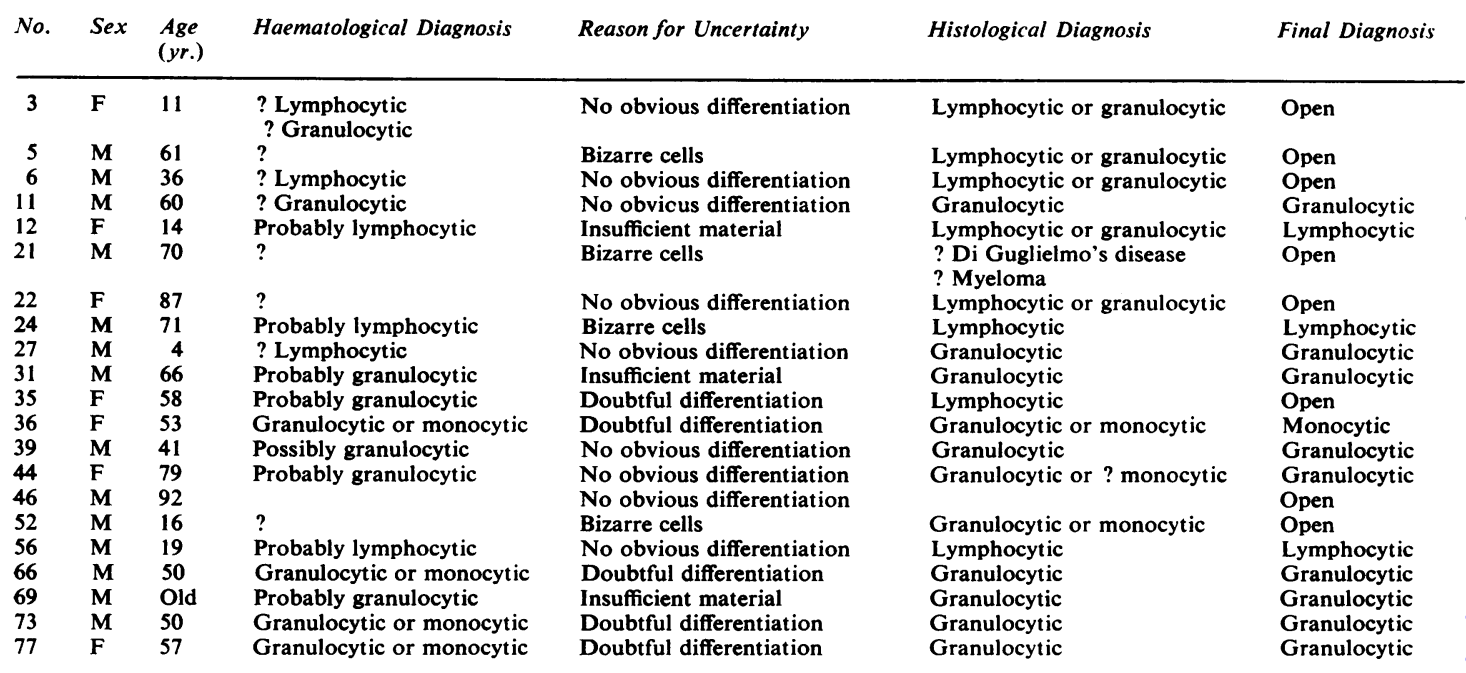

treatment, that a clear decision was very difficult. There was no apparent reason, on reviewing these cases, to prefer the histological to the haematological diagnosis in any one case of this group.

INITIAL HAEMATOLOGICAL DIAGNOSIS INDEFINITE In 21 cases $(31 \%)$ no certain diagnosis could be made as a result of the examination of haematological material. Ten of these were examined as unknowns on two separate occasions, and the diagnoses were either uncertain in both or they differed from each other. After comparison with the independent histological diagnosis and final review a decision on the type could be made in 13 of the 21 initially indefinite cases, but the diagnosis remained open in eight others. In Table III are shown the initial haematological and histological diagnoses, the reasons for the initial uncertainty, and the final diagnoses. In this table the entry 'no obvious differentiation' means that the blasts were themselves atypical (many showed nuclear lobe formation) and thus differed from the undifferentiated blasts which are 8 found in normal bone marrow, but that these $₹$ cellular changes did not progress towards anyo recognizable cell series. A special group was formed by cases $36,66,73$, and 77 in which a distinction between acute granulocytic and monocytic leukaemia had to be made. In each of them this proved impos- $\odot$ sible on the strength of the haematological appear- $N$ ances alone, but following review of the histological findings, definite diagnoses were made of granulo- 0 cytic leukaemia in three and of monocytic in one (case 36), in which there was considerable reticulin $\frac{0}{\Phi}$ formation in many sites. Table IV gives a summary $\stackrel{9}{\longrightarrow}$ of the diagnoses in all cases, and Table $I$ enumerates 0 the reasons for the diagnoses in the 48 cases finally classified as granulocytic.

CORRELATION OF CELlULAR TYPE WITH CLINICAL FEATURES Following our efforts to classify the cases 
TABLE IV

FINAL DIAGNOSES

\begin{tabular}{|c|c|c|c|}
\hline \multirow[t]{2}{*}{ Type } & \multicolumn{2}{|c|}{ Means of Making Initial Diagnosis } & \multirow[t]{2}{*}{ Total } \\
\hline & Haematological & Histological & \\
\hline Granulocytic & 39 & 9 & 48 \\
\hline Eosinophilic & 1 & - & 1 \\
\hline Di Guglielmo & 3 & - & 3 \\
\hline Lymphocytic & 4 & 3 & 7 \\
\hline $\begin{array}{l}\text { Monocytic } \\
\text { Open }\end{array}$ & - & 1 & $\begin{array}{l}1 \\
8\end{array}$ \\
\hline Total & 47 & 13 & 68 \\
\hline
\end{tabular}

of acute leukaemia it was important to ascertain if the types so established could be correlated with the clinical features. In this connexion we examined the incidence of certain symptoms and signs, as well as the patients' survival before and after the diagnosis of leukaemia was made.

The commonest symptoms mentioned in the records were lassitude and others arising from the presence of anaemia; fever, with or without obvious infections, and purpuric manifestations. The commonest abnormal physical signs were pallor, purpura, and splenomegaly. None of these showed a preference for any one cell type. Lymphadenopathy occurred in only 15 cases, in three of which it was confined to the neck and evidently secondary to a throat infection. It was relatively more common in the lymphocytic than in the granulocytic type $(57: 18 \%)$ but inconstant in both. Apart from this finding, it was evident that neither the nature of the symptoms nor of abnormal signs could be positively correlated with the cellular type, as diagnosed by us.

It is very difficult to gauge the duration of symptoms before the diagnosis is made. In many cases of acute leukaemia, especially in the older age groups, the onset of symptoms is exceedingly insidious, and their duration can only be guessed. When a definite event like fever or bleeding has shown forcibly that something is amiss, it often becomes clear in retrospect that lassitude and other vague signs pointing to a deterioration of health have been present for much longer periods. Accepting a stated duration of symptoms in excess of one month as denoting a relatively insidious mode of onset we found 28 of 48 patients with acute granulocytic leukaemia in this group $(60 \%)$, and 12 of 20 patients with all other forms $(60 \%)$. Among the seven patients accepted as belonging to the acute lymphocytic type there were three with an insidious onset. There was a slight difference in the duration of symptoms between young and older patients, the onset being insidious in six of $13(46 \%)$ aged up to 20 , against 34 of $55(62 \%)$ aged over 20 . This was not statistically significant. Among the patients classified as having granulocytic leukaemia were two with a history of polycythaemia vera and one with another myeloproliferative disorder, all with histories extending over several years.

Since a differential response to treatment according to cellular type has been found by a number of authors but denied by others, an attempt was made to obtain information on this point. This was done indirectly by correlating the survival time following diagnosis with the type of leukaemia. It has frequently been shown (Ellison, 1956; Freireich et al., 1961; Boggs et al., 1962) that survival in acute leukaemia is prolonged only if remissions are produced. It is therefore reasonable to interpret a relatively prolonged survival as indicating some response to therapy. The patients in our series were treated with corticosteroids and antimetabolites as well as ancillary means such as antibiotics and transfusions. The median survival in untreated acute leukaemia, both in adults and children, has been shown by Tivey (1954) to be approximately two months. We subdivided our patients into those surviving six months or more from diagnosis and those dying before that point. It seemed likely that the former group would encompass most of the patients responding in some measure to therapy.

Table $\mathrm{V}$ shows the survival times arranged by age groups and cellular types. It can be seen that of the 48 patients classified as suffering from acute granulocytic leukaemia only three survived in excess of six months, whereas there were six among the 20 patients with other types of acute leukaemia. This difference is statistically significant $\left(\chi^{2}=6.93, \mathrm{P}<0.01\right)$. On examining the age distribution of these patients, it became clear that the difference was much more striking among the youngest patients: among five under 20 with acute granulocytic leukaemia none lived longer than three months from diagnosis, but four of eight with other types lived in excess of six months. By contrast, in the group older than 20

\section{TABLE V}

SURVIVAL (DAYS) FROM DIAGNOSIS ACCORDING TO AGE AND CELL TYPE

\begin{tabular}{|c|c|c|}
\hline $\begin{array}{l}\text { Age of } \\
\text { Patients }\end{array}$ & Granulocytic & Other Types of Cell \\
\hline $0-10$ & 21,60 & 180,210 \\
\hline $11-20$ & $21,30,90$ & $3,30,90,120,250,365$ \\
\hline $21-30$ & 1,14 & - \\
\hline $31-40$ & $12,14,21$ & 1,180 \\
\hline $41-50$ & $1,3,30,45,60$ & \\
\hline $51-60$ & $1,2,3,12,14,14,60,120$ & $1,45,250$ \\
\hline $61-70$ & $\begin{array}{l}?, 1,1,3,6,30,30,30,45, \\
90,105,120,210\end{array}$ & 10,14 \\
\hline $71-80$ & $1,3,6,30,36,120,180,210$ & 10,14 \\
\hline $81-90$ & 6,45 & \\
\hline $\begin{array}{l}90+ \\
\text { 'Old' }\end{array}$ & -130 & 1 \\
\hline ‘Old' & 1,30 & - \\
\hline
\end{tabular}


years three of 43 with granulocytic and two of 12 with other types of acute leukaemia survived six months or more from diagnosis; the difference, though suggestive, is not statistically significant $\left(\chi^{2}=2 \cdot 67, \mathrm{P}<0 \cdot 1\right)$. It is of some interest that two of the three patients with Di Guglielmo's disease lived longer than six months; one was a boy of 11 , the other a woman of 58 .

\section{DISCUSSION}

In this investigation we set ourselves the twofold task of determining if we could classify acute leukaemias by routine pathological methods, and if so, whether the classification was reasonable when related to the clinical features.

The means of diagnosing the cellular type in acute leukaemia have been the subject of perennial controversy, the chief point at issue being whether or not those cells thought to be most typical of the disease, the leukaemic blasts, can themselves be recognized as occurring in more than one structurally or functionally distinctive form; if they can, acute leukaemia may be classified according to the morphology of the blasts; if not, other criteria, such as the nature of more mature accompanying cells, must be used. However, the latter cells need not belong to the same cell series as the blasts.

Methods of classifying blasts more subtle than a study of their morphology in conventionally stained films have been advocated, among them their appearance after peroxidase, supravital, or fluorescent staining, and in phase-contrast microscopy; their nucleolar formula, resistance to nucleases, growth pattern in tissue cultures, and others (Dameshek and Gunz, 1958; Hayhoe, 1960). Few of these are applicable in routine work and some are of doubtful validity. In general such methods may give reproducible results when applied to the classification of typical cells from normal tissues, but they are apt to fail to distinguish the grossly atypical blasts so often found in acute leukaemia.

Our own experinece showed that we could make an initial haematological diagnosis in nearly $70 \%$ of cases. In this connexion, most of the cellular characteristics of the blasts were of little help, since they were far too variable; this included nuclear chromatin pattern, cell size and shape, nuclear abnormalities, nucleolar numbers, and mitotic frequency. Nor did we find the accompanying mature cell forms helpful when the blasts could not be classified, since it was generally impossible to be certain whether both blasts and predominant mature cells belonged to the same series. We found much the most useful haematological criterion to lie in the presence or absence of cytoplasmic granules in at least some of the primitive cells; to this could $\stackrel{0}{F}$ be added that of Auer bodies which, being com- 을 posed of the same material as the neutrophil $\Rightarrow$ granules (Ackerman, 1950), have the same signi- $\frac{\text { P }}{+}$ ficance. The demonstration of the 'pseudo-Pelger' nuclear abnormality was also a clear means of 흠 distinguishing granulocytic cases; this abnormality $\frac{\hat{D}}{\vec{D}}$ is more widespread in acute leukaemia than has $\stackrel{\mathbb{2}}{\square}$ been realized. Apart from demonstrating it, and the is few clear cases of Di Guglielmo's disease, our $\vec{\circ}$ haematological examination was thus able to classify virtually only those cases in which the primitive cells $\vec{\omega}$ showed a tendency towards granule formation, and to separate them from others in which the early cells? showed no such tendency. A transition of blasts $\vec{\sigma}$ towards the lymphocytic series was noted in only a few instances, mostly in children.

Another approach to a classification of acute $\stackrel{\mathcal{G}}{\mathrm{S}}$ leukaemias is by way of their histological pattern. $\frac{}{5}$ We were concerned to see how often an independent ${ }_{\vec{C}}$ examination of the tissue would confirm the haematological diagnosis, and also whether it could provide a firm classification in cases where the haematolo- $\mathscr{\odot}$ gical findings were indefinite. We found that in $83 \%$. of the 47 haematologically diagnosed cases the histology was confirmatory, and that in $62 \%$ of the 21 haematologically indefinite cases the histological findings enabled a confident diagnosis to be made. In altogether 16 cases (eight each in the haematolo- $\stackrel{\Phi}{\Omega}$ gically 'definite' and 'indefinite' groups), or $24 \%$ of $\overrightarrow{\vec{O}}$ the total series, the histological examination was un- 3 helpful in making a diagnosis. This was mainly because by the time the patients came to necropsy, extensive tissue changes had occurred as a result of therapy.

Since the histological examinations in our series were carried out on post-mortem material, they could add nothing to the accuracy of diagnosis in $\frac{3}{3}$ the early stages of the disease. Since, however, we discovered characteristic changes in a large proportion of lymph nodes after death, it seems possible $\frac{D}{O}$ that biopsy of the nodes during life might have been found a useful additional method of distinction $N$ between cases of granulocytic and lymphocytic in leukaemia which could not be haematologically N diagnosed.

The eventual type distribution showed a heavy? preponderance of the granulocytic form, undoubted - 은 ly because of the unusual age distribution in ouros series (Table V). Had we been able to include $a^{-}$ higher proportion of children, the proportion of lymphocytic forms would almost certainly have been higher.

As it appears that we were able finally to give $a \stackrel{\mathbb{}}{a}$ name to nearly $90 \%$ of our cases of acute leukaemia the question arose in how far this helped in defining 
clinical types: could this classification be used in characterizing individual patients as to history, symptoms, signs, or response to therapy? We found few clinical differences between the groups but even in the small number of children in our series there was a clear difference in survival according to type; those classified as having granulocytic leukaemia lived for much shorter periods than the rest, and this would suggest that this group is relatively resistant to therapy by present-day methods. Similar findings have been obtained in larger series (Colebatch, 1961; Freireich et al., 1961 ; Thompson and Walker, 1962).

In adults it could not be shown that patients with the granulocytic type did less well than those with others, a finding similar to that of Freireich et al., 1961), although a difference might have been noted had our numbers been larger. Our results thus suggest that at least in children a division of acute leukaemia into types is of importance, in that a diagnosis of acute granulocytic leukaemia connotes a particularly bad prognosis.

We note in passing that we found a further factor which appeared to influence the prognosis in our series, namely, the height of the initial leucocyte count. Of 60 patients in whom this was known, 30 had counts up to 20,000 per c.mm. and 30 in excess of this figure. In the former group there were seven and in the latter only two survivals in excess of six months; the difference approached statistical significance $\left(\chi^{2}=3 \cdot 27 ; \mathbf{P}<0 \cdot 1\right)$. Several other authors have noted a similar finding (Zuelzer and Flatz, 1960; Haut et al., 1959; Freireich et al., 1961). Very high initial counts carried a particularly bad im- mediate prognosis. Of 10 patients with counts in excess of 100,000 per c.mm., only one, a child, lived more than two weeks from diagnosis; seven died within three days of admission, four of them from cerebral haemorrhage, a special risk in this high-count group, as pointed out by Fritz, Forkner, Freireich, Frei, and Thomas (1959) and by Freireich, Thomas, Frei, Fritz, and Forkner (1960).

\section{REFERENCES}

Ackerman, G. A. (1950). Blood, 5, 847.

Boggs, D. R., Wintrobe, M. M., and Cartwright, G. E. (1962). Medicine (Baltimore), 41, 163.

Colebatch, J. H. (1961). Med. J. Aust., 48, 624

Dameshek, W., and Gunz, F. (1958). Leukemia, pp. 93, 244. Grune and Stratton, New York and London.

Dorr, A. D., and Moloney, W. C. (1959). New Engl. J. Med., $261,742$.

Ellison, R. R. (1956). Med. Clin. N. Amer., 40, 743.

—, Gehan, E. A., Sulman, D., Boggs, D. R., and Frei, E., III (1961). J. chron. Dis., 14, 593.

Freireich, E. J., Thomas, L. B., Frei, E., III, Fritz, R. D., and Forkner, C. E. Jr. (1960). Cancer, 13, 146.

Fritz, R. D., Forkner, C. E. Jr., Freireich, E. J., Frei, E., III, and Thomas, L. B. (1959). New Engl. J. Med., 261, 59.

Goldberg, G. M. (1957). Lab. Invest., 6, 383.

- (1958). J. Anat. (Lond.), 92, 310.

, and Rubenstone, A. I. (1960). Cancer, 13, 513

- - - and Saphir, O. (1961a). Jbid., 14, 21.

, and Ungar, H. (1958). Lab. Invest., 7, 146.

Gunz, F. W., and Hough, R. F. (1956). Blood, 11, 882.

Haut, A., Altman, S. J., Wintrobe, M. M., and Cartwright, G. E. (1959). Blood, 14, 828.

Hayhoe, F. G. J. (1960). Leukaemia. Research and Clinical Practice, p. 73. Churchill, London.

Louis, J., Sanford, H. N., and Limarzi, L. R. (1958). J. Amer. med. Ass., 167, 1913.

Popper, H., and Schaffner, F. (1957). Liver: Structure and Function, p. 625. Blakiston, New York.

Thompson, R. B., and Walker, W. (1962). Brit. med. J., 1, 1165.

Tivey, H. (1954). Ann. N.Y. Acad. Sci., 60, 322.

Zuelzer, W. W., and Flatz, G. (1960). Amer. J. Dis. Child., 100, 886. 